УДК 543.51:543.53:553.96

\title{
АНАЛИТИЧЕСКИЕ ПОДХОДЫ К КОЛИЧЕСТВЕННОМУ ОПРЕДЕЛЕНИЮ СОДЕРЖАНИЙ ХИМИЧЕСКИХ ЭЛЕМЕНТОВ В УГЛЯХ И УГЛИСТЫХ ПОРОДАХ С ИСПОЛЬЗОВАНИЕМ МЕТОДОВ ИСП-МС И ИНАА
}

\author{
Зарубина Наталья Владимировна' \\ zarubina@fegi.ru
}

\author{
Блохин Максим Геннадьевич 1 , \\ blokhin@fegi.ru
}

Остапенко Дмитрий Сергеевич 1 , insunday888@gmail.com

\author{
Чекрыжов Игорь Юрьевич 1 , \\ chekr2004@mail.ru
}

\section{Арбузов Сергей Иванович2,} siarbuzov@mail.ru

\author{
Судыко Александр Федорович2, \\ sudykoAF@yandex.ru \\ 1 Дальневосточный геологический институт ДВО РАН, \\ Россия, 690022, г. Владивосток, пр. 100-летия Владивостока, 159. \\ 2 Национальный исследовательский Томский политехнический университет, \\ Россия, 634050, г. Томск, пр. Ленина, 30.
}

\begin{abstract}
Актуальность исследования обусловлена значительным интересом ученых, работающих в области угольной геологии, и специалистов химико-аналитических лабораторий, практикующих элементный количественный анализ объектов окружающей среды, к проблематике высокоточного определения низких содержаний химических элементов в углях и угольных материалах для последующего применения полученных данных при решении научных и различных технологических задач. Цель: рассмотреть и обобщить наиболее часто используемые методические подходы к определению химических элементов в широком диапазоне их содержаний в углях (угольных материалах), а также оценить правильность и точность результатов метода ИСП-МС после открытого кислотного разложения проб и их сплавления с метаборатом лития, сопоставлением получаемых данных с результатами метода ИНАА и статистической обработкой данных.

Объекты: угли, углистье алевролиты и углефицированная древесина месторождения германия Спецугли, расположенного на юго-западной окраине Ханкайского массива в восточной части Павловской угленосной впадины, Приморский край.

Методы: масс-спектрометрия с индуктивно-связанной плазмой (ИСП-МС), применённая после перевода исследуемых образцов в растворенную форму, и неразрушающий метод количественного инструментального нейтронно-активационного анализа (ИНАА). Для обработки полученных результатов были использованы методы математической статистики.

Результаты. Получены результаты ИСП-МС анализа трех стандартных образцов утвержденного типа (СО) состава золы уноса угля КАТЭКа ГСО 9237-2008 (ЗУК-2), золы бурого угля Азейского месторождения ГСО 7177-95 (ЗУА-1) и битуминозного угля CLB-1 Геологической службы США, представленные в виде данных об их микроэлементном составе в сравнении с аттестованными и рекомендованными значениями. Результаты определения части элементов, приведенные нами, дополняют представленные в паспорте данные по этим СО. Проведено сопоставление ИСП-МС результатов анализа для проб углей и углистых пород после различных способов химической пробоподготовки: методики открытого кислотного разложения и сплавления с метаборатом лития. Изучено поведение отдельных элементов во время этих процессов. Выполнено сравнение полученных данных по ИСП-МС и ИНАА методам. Все результаты обработаны метрологически, и показано их допустимое расхождение.
\end{abstract}

\section{Ключевые слова:}

Масс-спектрометрия с индуктивно связанной плазмой, инструментальный нейтронно-активационный анализ, стандартные образцы, химическая пробоподготовка, уголь, углистые породы, Спецугли, Павловская угленосная впадина.

\section{Введение}

По многочисленным исследованиям угли содержат большое количество химических элементовпримесей, в том числе ценных и токсичных [1,2 и др.], что делает сами угли и продукты их переработки потенциальным источником нетрадиционного сырья для извлечения ценных компонентов [3, 4 и др.]. В то же время в процессе сжигания (включая природные пожары) углей токсичные элементы могут попадать в окружающую среду, оказывая негативный эффект на ее экологическое состояние [5, 6 и др.]. Неорганические примеси в угле, отражающие его качественный состав, влияют на состояние технологического оборудования, задействованного в процессе переработки угля. Поэтому их содержание нормируется и должно контролироваться [7]. Изучение содержаний микро- 
элементов в углях важно в научном плане для геохимических исследований [8, 9 и др.]. Все это указывает на необходимость применения новых, усовершенствования и оценки существующих аналитических подходов к определению элементного состава углей и угольного сырья.

Угли по-прежнему считаются сложным объектом для анализа содержаний в них химических элементов. Это связано с трудностью выполнения пробоподготовки, спецификой угольной пробы и физикохимическими свойствами определяемых элементов. В настоящее время опубликовано большое количество работ, посвященных определению микроэлементного состава углей и продуктов их переработки [10, 11 и др.] с использованием различных аналитических методов. Данные методы можно подразделить на разрушающие, такие как плазменная спектрометрия (массспектрометрия с индуктивно-связанной плазмой (ИСП-МС) и атомно-эмиссионная спектрометрия с индуктивно-связанной плазмой (ИСП-АЭС)), атомноабсорбционная спектрометрия (ААС), и некоторые другие, неразрушающие (например, метод инструментального нейтронно-активационного анализа (ИНАА), рентгеновские методы анализа, электронную микроскопию). Все эти методы имеют свои преимущества и недостатки в проведении анализа. Матричные эффекты, наложения на аналитические сигналы определяемых элементов, присущие многим методам, могут увеличивать пределы обнаружения, а сложности, связанные с неоднородностью образца и, как следствие, представительностью пробы, в результате использования маленьких навесок для анализа (часто встречается при использовании неразрушающих методов) могут повлиять на точность получаемых результатов.

При проведении анализа с использованием методов ИСП-МС, ИСП-АЭС и ААС в классическом варианте пробы подвергают предварительному разложению и переводу определяемых компонентов в раствор. Данная процедура может быть сопряжена с рядом ограничений, которые отражаются на выборе способа пробоподготовки образцов к анализу. В результате нужно достичь следующих требований:

- количественного перехода элементов с различными химическими свойствами в растворенную форму;

- стабильности раствора во времени;

- предельно возможного уменьшения в растворе концентрации матричных элементов, оказывающих мешающее влияние на аналиты;

- недопущения загрязнения пробы анализируемыми химическими элементами на стадии пробоподготовки.

Для устранения этих сложностей в некоторых работах описываются приемы, когда при использовании разрушающих методов пробы не подвергаются предварительному химическому разложению. В частности, в методе электротермической атомно-абсорбционной спектрометрии (ЭТ-ААС) в графитовую кювету вносят порошкообразную пробу [12], электротермическое испарение твердой пробы применяют и в методе
ИСП-МС [13]. А применение лазерной абляции образца в данном методе является широко известным подходом [14, 15 и др.].

Следует подчеркнуть, что проблема корректного, с требуемой точностью определения элементовпримесей в металлоносных углях и продуктах их сжигания по-прежнему может возникать при анализе таких объектов. Многое в данном случае будет зависеть от технического оснащения лаборатории.

Тем не менее, как показывает практика, в случае валового анализа применение именно методов ИСПМС и ИСП-АЭС в микроэлементном анализе углей и продуктов их переработки, сопровождающееся предварительным химическим разложением проб, дает возможность определить максимальное количество химических элементов одновременно из одной пробы, с наиболее низкими пределами обнаружения и высокими метрологическими показателями получаемых результатов.

Метод ИНАА - мощный инструмент для определения элементного состава геологических образцов, в том числе и в углеродсодержащих со сложной матрицей. Его преимуществом является неразрушающий характер анализа, не требующий специальной пробоподготовки. Опубликован ряд работ, в которых методом ИНАА определены содержания микроэлементов в угольных объектах [16, 17 и др.]. Основной недостаток ИНАА - ограниченный спектр анализируемых элементов-примесей.

Имеется ряд опубликованных методических исследований по определению содержаний широкого спектра микроэлементов при изучении угольных материалов методом ИСП-МС [18, 19 и др.].

Для получения информации о химическом составе веществ методами плазменной спектрометрии, ввод исследуемых проб в ИСП в растворенной форме встречается наиболее часто в связи с такими преимуществами, как однородность поступающей пробы и удовлетворительная повторяемость результатов анализа. Поэтому определяющим фактором получения корректных результатов в данном случае является пробоподготовка. Обычно процедуры разложения угля включают сухое озоление [20], мокрое микроволновое озоление в закрытых сосудах [21], пирогидролиз [22, 23] и методы сжигания [24-26]. Могут использоваться и процедуры кислотной экстракции $[27,28]$.

Различные методы, предложенные Американским обществом по испытанию материалов (ASTM), рекомендуют стадию сухого озоления, за которой следует разложение золы смесью царской водки и дальнейшее растворение в азотной или фтористоводородной кислотах или, в некоторых случаях, сплавление образцов и растворение азотной кислотой или растворение в смеси соляно-плавиковой и борной кислот [29-31]. Российский ГОСТ Р 54237-2010 [32] (аналог ASTM D 6349-13 [30]) также регламентирует элементный анализ углей и продуктов их переработки, который выполняют либо после озоления образцов при $450-550{ }^{\circ} \mathrm{C}$ и последующего разложения с бора- 
тами лития, либо после разложения исходных проб с использованием минеральных кислот [18]. Однако при минерализации озолением в муфельной печи исключено корректное определение содержаний германия, селена, теллура, мышьяка, сурьмы, кадмия, образующиеся соединения которых летучи при таком способе подготовки проб. Наиболее привлекательным может быть способ кислотного разложения, при котором возможно определение большего числа элементов. Но и этот подход не лишен определенных трудностей.

В зависимости от минерального состава анализируемых образцов кислотное разложение проводят в закрытых или открытых системах. Такие, например, минералы, как кианит, циркон, топаз, силлиманит, могут быть разложены только в закрытых системах, т. е. в автоклавах с резисторным или микроволновым нагревах [33, 34], обеспечивающих интенсификацию процесса пробоподготовки образцов и исключение потери летучих соединений аналита. Но при использовании фтористоводородной кислоты HF, обязательного реагента для разрушения силикатной составляющей, необходимо удаление фторид-ионов, в противном случае будут занижены результаты определения редкоземельных элементов из-за образований нерастворимых соединений и соосаждения с фторидными смешанными солями кальция, магния, алюминия, натрия [35].

В случае использования закрытых систем для связывания фторид-ионов возможно применения борной кислоты $\mathrm{H}_{3} \mathrm{BO}_{3}$ [36]. Однако при этом увеличивается солевой фон и для последующего ИСП-МС измерения необходимо значительное разбавление анализируемых растворов, что может привести к понижению концентрации некоторых следовых элементов до уровней, находящихся ниже инструментального предела обнаружения. При применении открытых систем разложения для удаления избытка $\mathrm{SiF}_{4}$ и $\mathrm{HF}$ используют несколько последовательных упариваний аналита с минеральной кислотой, что дает возможность полностью избавиться от фторид-ионов и растворить смешанные фторсодержащие соли [37, 38]. В этом случае устранение из образца силикатной составляющей уменьшает нагрузку матричных компонентов на ИСП прибор и определяет наиболее низкие пределы обнаружения следовых элементов.

Методы сжигания, в качестве альтернативы, считаются подходящими для разложения угля из-за относительно малого расхода реагентов и высокой эффективности разложения проб [39]. Однако при использовании колб с током кислорода в качестве систем сжигания навеска образца обычно ограничивается примерно 0,15 г [24]. Методы, основанные на реакциях горения с кислородом, имеют преимущество превращения органических материалов в соответствующие продукты горения с использованием только этого газа, что сводит к минимуму риск загрязнения $[39,40]$. В качестве примера применения подобной методики В. Ген и соавторы [24] использовали метод сжигания в кислородной колбе для разложения угля и дальнейшего определения содержания серы и ртути с помощью ИСП-АЭС. Для абсорбции ртути использовался раствор $\mathrm{KMnO}_{4}$, максимальная навеска образца, которая могла быть разложена по данной схеме, составляла 0,11 г. В. Диас и М. Сатте [41] использовали бомбу сгорания для разложения угля и других проб окружающей среды для последующего анализа с помощью ЭТ-ААС. Предлагаемая процедура занимала много времени, кроме того одновременно нельзя было подвергать пробоподготовке несколько образцов.

Следует выделить ряд работ, в которых представлены методические исследования с использованием микроволнового (МВ) разложения и ИСП-МС определений.

Мокрое МВ разложение широко применяется для процедуры пробоподготовки ввиду его высокой эффективности по отношению к большинству образцов различного состава, относительно низкого расхода реагентов и снижения рисков потерь и загрязнения по сравнению с обычными процедурами разложения [42]. Однако даже при использовании концентрированных кислот, высокой температуры и давления сообщалось о неполном вскрытии угля [43]. С другой стороны, одна из возможных проблем при МВё разложении это максимальная навеска образца, обычно не превышающая 0,2 г, что затрудняет получение низких пределов обнаружения и воспроизводимых результатов [20, 43].

В публикации [15] предложено четыре варианта пробоподготовки: МВ разложение углей с использованием смеси $\mathrm{HNO}_{3}$ и $\mathrm{H}_{2} \mathrm{O}_{2}$; $\mathrm{HNO}_{3}$ и $\mathrm{H}_{2} \mathrm{O}_{2}$ с добавлением HF; царской водки; царской водки с добавлением HF. Авторы пришли к выводу, что наиболее подходящим способом кислотного микроволнового вскрытия является вариант применения смеси $\mathrm{HNO}_{3}$ и $\mathrm{H}_{2} \mathrm{O}_{2}$ с добавлением HF. Для оценки точности был проанализирован стандартный образец $(\mathrm{CO})$ состава угля битуминозного SRM1635. Получены результаты с удовлетворительной точностью по 22 из 25 аттестованным для стандарта элементам. Также были проведены исследования по разложению образцов угля методом сплавления с метаборатом лития $\mathrm{LiBO}_{2}$. Сплавление дает максимальную полноту перехода в раствор таких элементов, как $\mathrm{Si}, \mathrm{Cr}, \mathrm{Y}, \mathrm{Zr}$, Hf и $\mathrm{W}$, однако при этом наблюдались потери $\mathrm{S}, \mathrm{Cu}, \mathrm{Zn}, \mathrm{As}$, $\mathrm{Se}, \mathrm{Ag}, \mathrm{Cd}, \mathrm{Te}, \mathrm{Sn}, \mathrm{I}, \mathrm{Re}, \mathrm{Pb}, \mathrm{Hg} \mathrm{Tl}$ и Bi. Измерение концентрации 70 элементов выполнено в сочетании ИСП-АЭС и ИСП-МС высокого разрешения.

В исследовании [44] представлена экспрессная методика подготовки образцов угля к анализу методом ИСП-МС с применением МВ разложения и использованием смеси $\mathrm{HNO}_{3}-\mathrm{H}_{2} \mathrm{O}_{2}$. Было выполнено ИСП-МС определение $\mathrm{As}, \mathrm{Ba}, \mathrm{Co}, \mathrm{Cs}, \mathrm{Cu}, \mathrm{Ga}, \mathrm{Ge}, \mathrm{Mn}, \mathrm{Ni}, \mathrm{V}, \mathrm{Pb}$, $\mathrm{Sr}, \mathrm{Zn}, \mathrm{Zr}$ и U. Отмечено, что при использовании предложенной методики разложения результаты определения Ti и Hf были занижены. Для остальных элементов были получены удовлетворительные результаты. Предел обнаружения элементов составил 0,001-0,57 мкг/г (кроме Ті, для него - 4 мкг/г). Относительное стандартное отклонение не превышало 3,5 \%. Для контроля точности результатов определения были использованы стандартные образцы угля SRM 18, 19, 20. 
В работе [45] для определения урана в угольной золе уноса было также предложено использовать ИСП-МС метод в сочетании с МВ разложением. Перевод образца в аналитическую форму осуществлялся следующим образом: навеска обрабатывалась в МВ печи смесью $\mathrm{HNO}_{3}$, $\mathrm{HF}$ и $\mathrm{HClO}_{4}$. Заявленный предел обнаружения $-0,05$ мкг/г, воспроизводимость результатов от 95 до $104 \%$, а относительное стандартное отклонение для шести результатов составило $3,2 \%$.

Процедура МВ разложения проб углей была задействована при определении распределения микроэлементов в углях с помощью последовательного химического выщелачивания (кислотной экстракции) [28]. Данная методика предполагала поэтапное воздействие химических реагентов на навеску угольного материала с целью установления привязки того или иного химического элемента к определенной органической/неорганической фазе угля. МВ воздействие на пробу было использовано в данном эксперименте для разложения органической и силикатной составляющих пробы. Растворы после выщелачивания анализировались при помощи ИСП-МС и ИСП-АЭС методов.

Использование закрытых МВ систем дает несколько преимуществ, включая удержание «летучих» элементов, меньшие временные затраты на пробоподготовку. Но имеются и определенные трудности, поскольку органическая матрица не может быть разложена полностью с помощью реагентов, обычно применяемых в МВ системах. Для полного разложения необходимо предварительно выполнять дополнительные процедуры, например, окислительный пиролиз. Такой подход к разложению, основанный на окислительном пиролизе и $\mathrm{MB}$ разложении для количественного определения как основных, так и ультрамалых по содержанию элементов методами плазменной спектрометрии, был предложен в [46]. Образцы угля были первоначально подвергнуты пиролизу при нагревании до $\sim 500{ }^{\circ} \mathrm{C}$ в потоке кислорода. Затем остаток был обработан в МВ системе с помощью смеси $20 \% \mathrm{HNO}_{3}+5 \% \mathrm{HF}+5 \% \mathrm{H} 2 \mathrm{O} 2$. Таким образом, время на пробоподготовку увеличивается, она усложняется, и появляется необходимость использования дополнительной установки для пиролиза.

В литературе приводится еще один комплексный подход с процедурой МВ разложения и предварительного термического воздействия на пробу. Возможность МВ сжигания и его преимущества по сравнению с традиционными методами сжигания были продемонстрированы для полного разложения органических образцов в закрытых сосудах $[47,48]$. Этот метод сочетает в себе черты классических методов сжигания с особенностями систем МВ разложения. Методика включает сжигание образцов в закрытых кварцевых сосудах под давлением кислорода и дожигание под воздействием МВ излучения. Дополнительная стадия дефлегмации обеспечивала надежное растворение оставшихся неорганических соединений и позволяла количественно извлекать аналиты $[49,50$ и др.]. В данном случае лучше избегать использования концентрированных кислот, и для большинства аналитов можно использовать их в разбавленном ви- де. Эту процедуру можно считать безопасной, поскольку она выполняется в специальной МВ печи и обеспечивает относительно высокую пропускную способность. Кроме того, остаточное содержание углерода получалось чрезвычайно низким, что позволяло избежать дополнительных помех в методах измерения. Эта методика показала возможность ее применения для разложения угля и дальнейшего определения галогенов с использованием карбоната аммония $\left(\mathrm{NH}_{4}\right)_{2} \mathrm{CO}_{3}$ в качестве абсорбирующего раствора с хорошими результатами [25]. Возможности ее использования при анализе угля для определения металлов и металлоидов была продемонстрирована в работе [51]. Была предложена методика МВ сжигания для разложения угля и последующего определения $\mathrm{As}, \mathrm{Cd}, \mathrm{Hg}$ и $\mathrm{Pb}$. Были исследованы рабочие параметры, такие как вид абсорбирующего раствора и использование дополнительной стадии дефлегмации. Точность оценивалась с использованием сертифицированных $\mathrm{CO}$ и приемов по оценке воспроизводимости получаемых результатов для аналитов. Предложенная методика применялась для разложения проб угля с различной зольностью. $\mathrm{As}, \mathrm{Cd}$ и $\mathrm{Pb}$ определяли с помощью ИСП-МС, a $\mathrm{Hg}$ - с помощью ИСП-МС с «холодным паром». Для сравнения результатов измерения были выполнены с помощью ИСП-АЭС, в том числе и в варианте «холодного пара» для определения Нg. На наш взгляд, данная методика, несмотря на все ее преимущества, все же может иметь ограниченное применение в аналитической практике из-за некоторой «громоздкости» ее аппаратного оформления. В этой связи мы считаем рациональным использование аналитической схемы пробоподготовки способом открытого кислотного разложения смесью $\mathrm{HNO}_{3}, \mathrm{HClO}_{4}$ и $\mathrm{HF}$ для образцов углей с зольностью менее $30 \%$ и сплавления золы угля с $\mathrm{LiBO}_{2}$ в случае зольности более $30 \%$. Такой подход подробно описан в работе Г.А. Олейниковой с соавторами [18].

Ранее нами была использована методика ИСП-МС определения рения после открытого кислотного разложения углеродсодержащих, органоминеральных и органогенных геологических образцов $[52,53]$. Для выполнения анализа углей методика была оптимизирована [54]: обработку концентрированными кислотами-окислителями $\mathrm{HNO}_{3}$ и $\mathrm{HClO}_{4}$ проводили трижды до полного разрушения органического вещества.

\section{Материалы и методы}

В настоящей работе были проанализированы более 40 проб углей, углистых алевролитов и углефицированной древесины месторождения германия Спецугли, расположенного на юго-западной окраине Ханкайского массива в восточной части Павловской угленосной впадины, Приморский край. Результаты сравнительного анализа методами ИСП-МС с различными способами пробоподготовки и сопоставление данных ИСП-МС и ИНАА определения показаны на примере двух проб: пробы СУ-18-18 (зольность $5,3 \%)$, относящейся к углю, и углистому алевролиту СУ-7-18 (зольность 65,0 \%). Особенности геологического строения и металлоносности месторождения 
германия Спецугли достаточно детально описаны во многих работах [55, 56 и др]. Угленосные отложения на месторождении представлены серыми и коричневато-серыми слаболитифицированными аргиллитами, алевролитами и песчаниками с линзами и горизонтами галечников и включениями углефицированной древесины. Повышенные содержания Ge относятся к местному возвышению гранитного фундамента с примыкающими и перекрывающими его четырьмя рудоносными угольными пластами (I, II нижний, II верхний, III нижний) павловской свиты эоценолигоценового возраста с общей мощностью угленосных отложений до 100 м. Угли месторождения относятся к бурым, подгруппы 2БВ, среднезольные (16-18\%), малосернистые $(0,4-0,5 \%)$, с теплотой сгорания «рабочего» топлива 12,0-12,5 МДж/кг. УГлистые алевролиты отличаются повышенным содержанием минерального вещества и соответственно высокой зольностью. Углефицированная древесина (угольные включения) представлена остатками стволов и веток в межугольных слаболитифицированных песчаниках, часто аномально обогащена широким спектром редких элементов.

Анализ проб методом ИСП-МС выполнялся в ЦКП «Приморский центр локального элементного и изотопного анализа» Дальневосточного геологического института ДВО РАН, г. Владивосток.

Для пробоподготовки исследуемых образцов углей способом открытого кислотного разложения и последующего измерения концентрации элементов методом ИСП-МС была выбрана аналитическая схема с использованием азотной, хлорной и фтористоводородной кислот. При таком разложении происходит разрушение органической матрицы и силикатной составляющей и выделение $\mathrm{Si}$ в виде летучего фторида кремния $\mathrm{SiF}_{4}$, что приводит к его фактическому удалению из пробы.

Химическая пробоподготовка. Навески проб массой 0,05 г помещали в тефлоновые бюксы, добавляли $2 \mathrm{~cm}^{3} \mathrm{HNO}_{3}, 1 \mathrm{~cm}^{3} \mathrm{HClO}_{4}$ и давали выстояться 12 часов при комнатной температуре. Затем содержимое бюксов упаривали при температуре $140-150{ }^{\circ} \mathrm{C}$ до влажных солей. Эту процедуру выполняли дважды. К оставшейся части вещества приливали $2 \mathrm{~cm}^{3} \mathrm{HNO}_{3}$, $0,5 \mathrm{~cm}^{3} \mathrm{HClO}_{4}$ и $2 \mathrm{~cm}^{3} \mathrm{HF}$ («suprapur», Merck), упаривали до состояния влажных солей. Для удаления избытка HF и разрушения образовавшихся в ходе разложения пробы фторсодержащих солей выполняли двукратное упаривание аналита с азотной кислотой (1:1). После этого в бюксы вносили по $10 \mathrm{~cm}^{3} 10 \%$ $\mathrm{HNO}_{3}$, нагревали до перехода солей в растворенную форму. Полученные растворы переносили в полипропиленовые мерные колбы объемом $50 \mathrm{~cm}^{3}$ и доводили их деионизированной водой (тип I) до метки с добавлением следов HF для устранения возможности полимеризации и гидролиза высокозарядных ионов элементов Zr, Nb, Hf, Ta, а также Мо и W. Перед ИСП-МС анализом растворы проб разбавляли таким образом, чтобы конечный фактор разбавления составил 2500 .
Для выполнения ИСП-МС анализа золы углей пробы угольных материалов предварительно озоляли при температуре $550 \pm 10{ }^{\circ} \mathrm{C}$. Навески массой 0,05 г золы угля сплавляли в платиновых тиглях с метаборатом лития в пропорции 1:3 при температуре $1050{ }^{\circ} \mathrm{C}$ в течение 15 минут. Методика подготовки проб золы углей сплавлением с метаборатом лития и последующей стабилизацией полученных растворов нами подробно описана в [57].

Bсе используемые для выполнения химической пробоподготовки реактивы были высокой степени чистоты. Азотную кислоту марки «осч» перегоняли на установке перегонки без кипения фирмы MileStone, плавиковая, хлорная кислоты и метаборат лития были квалификации «suprapur». Деионизированную воду с удельным сопротивлением $18,2 \mathrm{M} \Omega / \mathrm{cm}$ получали с помощью системы очистки воды MiliQ, Millipore.

ИСП-МС определение микроэлементного состава проб углей и их зол проводилось на массспектрометрах фирмы Agilent, Япония. Образцы после открытого кислотного разложения анализировались на ИСП-МС модели 7700x, после разложения по методике сплавления с метаборатом лития - на модели 7500с. Оба инструмента использовались в режиме коррекции фона с помощью наполняемой гелием октопольной столкновительной ячейки с дискриминацией по энергии (ORC технология).

ИНАА был выполнен на исследовательском ядерном реакторе ИРТ-Т ИШЯТ Томского политехнического университета (ТПУ). Реактор был сконструирован и запущен в ТПУ в 1967 г. Реактор ИРТ-Т представляет собой многопрофильный объект и широко используется для фундаментальных и прикладных исследований в области ядерной и нейтронной науки и техники. Реактор ИРТ-Т имеет 10 горизонтальных экспериментальных каналов (ГЭК) и 14 вертикальных (ВЭК). Два вертикальных канала с внутренним диаметром 32 мм установлены в центре активной зоны реактора. При работе реактора на мощности 6 Мвт в них достигается максимальная плотность потока тепловых нейтронов $1,7 \cdot 10^{14} \mathrm{Hc} / \mathrm{cm}^{2}$. Двенадцать других ВЭК расположены за пределами центральной зоны и имеют диаметр до 55 мм. Средняя величина потока тепловых нейтронов в них составляет $2-5 \cdot 10^{13} \mathrm{Hc} / \mathrm{cm}^{2}$.

Анализ проводился в постоянном вертикальном канале, закрепленном за Ядерно-геохимической лабораторией, тепловыми нейтронами с интегральным потоком $2 \cdot 10^{17}-1,5 \cdot 10^{18} \mathrm{H} / \mathrm{cm}^{2}$. Масса навески образцов угольных материалов составляла от 100 до 300 мг. Приготавливалось по шесть параллельных навесок для каждого образца. Пробы заворачивались в алюминиевую фольгу известного состава. Времена облучения и охлаждения определялись опытным путем, а также основываясь на фактическом содержании анализируемых элементов в исследуемых пробах. Исходя из технических возможностей используемого оборудования определялись аналитические гамма-линии изучаемых элементов, когда влияние мешающих фотопиков минимально. Более полно методика и инструментальная часть описаны в [58]. 


\section{Результаты и их обсуждение}

Наши исследования посвящены определению микроэлементного состава угольных материалов, поскольку определение макроэлементного состава достаточно полно представлено в уже существующих методиках. Сведения же по их микроэлементному составу не всегда отражают широкий спектр элементов. Кроме того, существует проблема оценки корректности полученного результата измерений. Для этой цели используются СО элементного состава углей и углистых пород. Тем не менее их применение для оценки точности анализа содержаний следовых элементов зачастую затруднено из-за отсутствия аттестованных характеристик. Эта ситуация имеет место и при оценке качества результата элементоопределений для угольных материалов. Наиболее полно по микроэлементному составу охарактеризованы СО углей Геологической службы США SARM18, SARM 19 и SARM20, но они не отражают всего многообразия по составу имеющихся в природе углей. Доступный в аналитической практике российских лабораторий СО битуминозного угля CLB-1 Геологической службы США аттестован на незначительное число микроэлементов (аттестовано 10 показателей и 13 приведено в статусе рекомендованных).

По предлагаемой нами методике с использованием открытого кислотного разложения и измерения концентраций элементов методом ИСП-МС был выполнен анализ данного СО в условиях воспроизводимости (другое разложение, новый аналитик, выполнение измерений по разным дням в течение длительного периода времени и т. д.) Результаты измерений, их метрологическая оценка [59] и сравнение с паспортными данными приведены в табл. 1. Сравнение полученных данных ИСП-МС измерений с аттестованными и рекомендованными содержаниями элементов в СО показывает их удовлетворительную согласованность. Также проведенный анализ СО позволил получить данные по содержанию 23 элементов, которые отсутствуют в паспорте образца. Содержания (мкг/г) для них составили: Ве - 1,09 $\pm 0,21 ; \mathrm{Ge}-9,80 \pm 1,26$; $\mathrm{Sr}-93,0 \pm 21,1 ; \mathrm{Y}-4,71 \pm 0,71 ; \mathrm{Zr}-9,56 \pm 2,93$; $\mathrm{Cd}-0,13 \pm 0,04 ; \mathrm{Sn}-0,76 \pm 0,47 ; \mathrm{Cs}-0,29 \pm 0,03$;

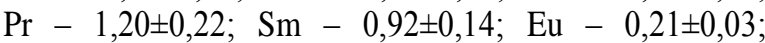
$\mathrm{Gd}-0,93 \pm 0,14 ; \mathrm{Tb}-0,14 \pm 0,02 ;$ Dy - 0,81 $\pm 0,08$

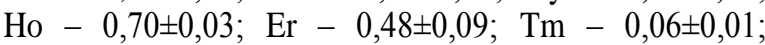
$\mathrm{Yb}-0,42 \pm 0,06 ; \mathrm{Lu}-0,06 \pm 0,02 ; \mathrm{Hf}-0,27 \pm 0,04$; $\mathrm{Ta}-0,09 \pm 0,02 ; \mathrm{W}-0,67 \pm 0,17$ и $\mathrm{Tl}-0,66 \pm 0,09$.

Помимо открытого кислотного разложения непосредственно исходных проб углей в работе была использована методика анализа золы после сплавления с $\mathrm{LiBO}_{2}$ и сравнением получаемых результатов. Оценка правильности определения элементов в золе углей проведена с использованием СО состава золы уноса угля КАТЭКа ГСО 9237-2008 (ЗУК-2) и золы бурого угля Азейского месторождения ГСО 7177-95 (ЗУА-1). Результаты ИСП-МС определения элементов для СО зол в сопоставлении с аттестованными и рекомендованными значениями приведены в табл. 2. Помимо этого, результаты измерения некоторых элементов в СО 3УА-1, приведенные нами, дополняют представленные в паспорте данные по этому образцу.

Таблица 1. Результаты ИСП-МС определения элементов (мкг/2) в СО угля CLB-1

Table 1. Results of ICP-MS determination of elements $(\mu \mathrm{g} / \mathrm{g})$ in a coal CRM CLB-1

\begin{tabular}{|c|c|c|}
\hline \multirow[b]{2}{*}{$\begin{array}{c}\text { Элементы } \\
\text { Elements }\end{array}$} & \multicolumn{2}{|r|}{ CLB-1 } \\
\hline & $\begin{array}{c}\text { Найдено X, } \\
(\mathrm{n}=20) \\
\text { Found X, } \\
(\mathrm{n}=20)\end{array}$ & $\begin{array}{c}\text { Аттестованные и рекомендо- } \\
\text { ванные* значения } \\
\text { Certified and recommended* } \\
\text { values }\end{array}$ \\
\hline $\mathrm{Li}$ & $7,26 \pm 1,43$ & $8 *$ \\
\hline $\mathrm{Sc}$ & $2,06 \pm 0,41$ & $2,0 \pm 0,1$ \\
\hline $\mathrm{V}$ & $13,1 \pm 3,6$ & $12 \pm 1$ \\
\hline $\mathrm{Cr}$ & $9,90 \pm 1,50$ & $9,7 \pm 1,2$ \\
\hline $\mathrm{Co}$ & $6,34 \pm 0,96$ & $7 \pm 0,7$ \\
\hline $\mathrm{Ni}$ & $19,7 \pm 3,9$ & $18 \pm 2$ \\
\hline $\mathrm{Cu}$ & $13,1 \pm 4,2$ & $10^{*}$ \\
\hline $\mathrm{Zn}$ & $49,5 \pm 5,8$ & $48 \pm 4$ \\
\hline $\mathrm{Ga}$ & $2,99 \pm 0,58$ & $3 *$ \\
\hline As & $13,6 \pm 1,2$ & $13^{*}$ \\
\hline $\mathrm{Se}$ & $2,03 \pm 0,51$ & $2 *$ \\
\hline $\mathrm{Rb}$ & $5,00 \pm 0,17$ & $5,2 \pm 0,9$ \\
\hline $\mathrm{Nb}$ & $1,18 \pm 0,20$ & $1 *$ \\
\hline Mo & $10,9 \pm 1,5$ & $9 *$ \\
\hline $\mathrm{Sb}$ & $1,36 \pm 0,07$ & $1,5^{*}$ \\
\hline $\mathrm{Ba}$ & $43,3 \pm 9,7$ & $34 \pm 5$ \\
\hline $\mathrm{La}$ & $4,88 \pm 0,90$ & $5 *$ \\
\hline $\mathrm{Ce}$ & $10,2 \pm 2,06$ & $10 \pm 1,6$ \\
\hline $\mathrm{Nd}$ & $4,49 \pm 0,50$ & $5^{*}$ \\
\hline $\mathrm{Pb}$ & $5,68 \pm 1,47$ & $5,1 \pm 0,7$ \\
\hline Th & $1,24 \pm 0,17$ & $1,4 *$ \\
\hline $\mathrm{U}$ & $0,47 \pm 0,04$ & $0,55^{*}$ \\
\hline
\end{tabular}

Была проведена сравнительная оценка двух способов пробоподготовки для метода ИСП-МС, заключающихся в открытом кислотном разложении и сплавлении проб с $\mathrm{LiBO}_{2}$. Полученные данные по результатам анализа золы проб углей методом сплавления были пересчитаны на уголь с учетом зольности и сопоставлены с результатами анализа исходных проб СУ-18-18 и СУ-7-18 по методике открытого кислотного разложения. Результаты определения содержаний элементов в двух из более 40 проанализированных проб с использованием двух способов разложения представлены в табл. 3, где $\mathrm{S}_{\mathrm{R}}$, мкг/г- стандартное отклонение в условиях воспроизводимости, a $\mathrm{RSD}_{\mathrm{R}}, \%$ - относительное стандартное отклонение в этих же условиях.

При выполнении анализа золы углей исключено достоверное определение германия, селена, теллура, мышьяка, сурьмы, образующиеся соединения которых летучи, при таком способе подготовки проб. Определение их содержания необходимо выполнять только после кислотного разложения. Вместе с тем в углях можно выделить такие элементы, как $\mathrm{Zr}, \mathrm{Hf}, \mathrm{Nb}$, Ta, Sn, определение которых после кислотного разложения может привести к заниженным результатам с увеличением зольности. Это связано с увеличением вклада минеральной составляющей углей: принимает другой вид соотношение форм нахождения элементов, возрастает доля трудноразлагаемых оксидов и силикатных минералов. В работе [18] отмечено, что для этих элементов полнота вскрытия проб с зольностью 
выше 30 \% возможна только при сплавлении золы угля и углистых пород с метаборатом лития. Это отчетливо прослеживается по результатам анализа проб, приведенных в табл. 3.

При ИСП-МС анализе углей после их озоления и последующего сплавления с $\mathrm{LiBO}_{2}$ нами было отмечено, что результаты определения $\mathrm{Cu}$ и $\mathrm{Ni}$ зачастую получаются завышены, по сравнению с методикой подготовки образцов открытым кислотным разложением (табл. 3). В настоящее время дать однозначного ответа о природе этого явления мы не можем. Как рабочие версии рассматриваются матричные эффекты при проведении ИСП-МС измерений, возможное увеличение инструментального предела обнаружения в результате воздействия больших концентраций $\mathrm{LiBO}_{2}$ на металлические пробоотборные конуса прибора. Постоянный контроль «холостых» проб в процессе анализа не позволил нам также сделать окончательного вывода о причинах данного факта. Наблюдения в этом направлении будут нами продолжены. Но на данный момент для точного определения $\mathrm{Cu}$ и Ni для нас предпочтителен метод кислотной минерализации проб.

Что касается определения лантаноидов, то значительных расхождений при использовании двух способов разложения не выявлено. Относительное стандартное отклонение $\left(\mathrm{RSD}_{\mathrm{R}}\right)$ не превышает $30 \%$, что хорошо согласуется с требованиями к аналитическим результатам при геохимических исследованиях $[60,61]$.

Для сравнительной оценки результатов определения элементов в угольных материалах был выполнен анализ одних и тех же образцов методами ИСП-МС после открытого кислотного разложения проб и ИНАА. Результаты определения и оценка относительного расхождения $\left(\mathrm{D}_{\mathrm{R}}, \%\right)$ по двум пробам показаны в табл. 4.

Таблица 2. Результаты ИСП-МС определения элементов (мкг/2) в СО золь уноса угля ЗУК-2 и золь бурого угля ЗУА-1

Table 2. Results of ICP-MS determination of elements $(\mu \mathrm{g} / \mathrm{g})$ in CRMs of fly coal ash ZUK-2 and brown coal ash ZUA-1

\begin{tabular}{|c|c|c|c|c|}
\hline \multirow{2}{*}{$\begin{array}{c}\text { Элементы } \\
\text { Elements }\end{array}$} & \multicolumn{2}{|c|}{ 3УК-2/ZUK-2 } & \multicolumn{2}{|c|}{ 3УA-1/ZUA-1 } \\
\hline & $\begin{array}{l}\text { Найдено } \mathrm{X},(\mathrm{n}=8) \\
\text { Found } \mathrm{X},(\mathrm{n}=8)\end{array}$ & $\begin{array}{l}\text { Аттестовано } \\
\text { Cert. Values }\end{array}$ & $\begin{array}{l}\text { Найдено X, }(\mathrm{n}=6) \\
\text { Found X, }(\mathrm{n}=6)\end{array}$ & $\begin{array}{l}\text { Аттестовано } \\
\text { Cert. Values }\end{array}$ \\
\hline $\mathrm{Be}$ & $2,85 \pm 0,41$ & $2,9 \pm 0,5$ & $10,7 \pm 1,1$ & $11 \pm 0,2$ \\
\hline Sc & $9,42 \pm 1,11$ & $8,6 \pm 0,9$ & $29,2 \pm 2,9$ & $27 \pm 5$ \\
\hline $\mathrm{V}$ & $66,8 \pm 2,2$ & $63 \pm 7$ & $146 \pm 4$ & $145 \pm 15$ \\
\hline $\mathrm{Cr}$ & $43,9 \pm 5,6$ & $42 \pm 4$ & $99,5 \pm 3,3$ & $99 \pm 8$ \\
\hline $\mathrm{Co}$ & $30,9 \pm 4,2$ & $26 \pm 2$ & $28,2 \pm 5,7$ & $25 \pm 4$ \\
\hline $\mathrm{Ni}$ & $64,4 \pm 8,2$ & $68 \pm 8$ & $64,7 \pm 8,8$ & $66 \pm 10$ \\
\hline $\mathrm{Cu}$ & $50,5 \pm 9,6$ & $51 \pm 7$ & $156 \pm 24$ & $176 \pm 18$ \\
\hline $\mathrm{Zn}$ & $71,0 \pm 7,2$ & $76 \pm 6$ & $66,5 \pm 4,9$ & $77 \pm 13$ \\
\hline $\mathrm{Ga}$ & $18,5 \pm 4,0$ & $15 \pm 3$ & $22,1 \pm 2,5$ & $21^{*}$ \\
\hline $\mathrm{Rb}$ & $12,3 \pm 2,5$ & $13,0 \pm 3,0$ & $22,1 \pm 4,2$ & $22 \pm 9$ \\
\hline $\mathrm{Sr}$ & $8259 \pm 424$ & $8300 \pm 700$ & $402 \pm 8$ & $403 \pm 33$ \\
\hline $\mathrm{Y}$ & $16,3 \pm 2,2$ & $15 \pm 6$ & $91,3 \pm 5,4$ & $87 \pm 17$ \\
\hline $\mathrm{Zr}$ & $138 \pm 19$ & $130 \pm 20$ & $317 \pm 58$ & $330 \pm 20$ \\
\hline $\mathrm{Nb}$ & $7,23 \pm 1,22$ & $7,8 \pm 1,1$ & $30,7 \pm 4,6$ & $34 \pm 6$ \\
\hline Mo & $2,55 \pm 0,66$ & $3,0 \pm 0,9^{*}$ & $7,22 \pm 0,36$ & $7,4 \pm 1,3$ \\
\hline $\mathrm{Sn}$ & $2,72 \pm 0,58$ & $3,2 \pm 0,2 *$ & $9,66 \pm 1,48$ & $11 \pm 2$ \\
\hline $\mathrm{Ba}$ & $8774 \pm 436,0$ & $8600 \pm 900$ & $263 \pm 4$ & $280 \pm 40$ \\
\hline $\mathrm{La}$ & $20,9 \pm 1,4$ & $20 \pm 2$ & $70,3 \pm 12,8$ & $70 \pm 10$ \\
\hline $\mathrm{Ce}$ & $39,7 \pm 2,4$ & $37 \pm 5$ & $141 \pm 12$ & $138 \pm 25$ \\
\hline $\operatorname{Pr}$ & $4,28 \pm 0,41$ & $4,2 \pm 0,4^{*}$ & $17,4 \pm 1,9$ & н.д./n.d. \\
\hline $\mathrm{Nd}$ & $15,6 \pm 2,6$ & $17 \pm 1$ & $70,0 \pm 6,1$ & н.д./n.d. \\
\hline $\mathrm{Sm}$ & $3,03 \pm 0,51$ & $3,2 \pm 0,2$ & $15,5 \pm 1,7$ & $15,0^{*}$ \\
\hline $\mathrm{Eu}$ & $0,96 \pm 0,12$ & $0,8 \pm 0,2 *$ & $2,23 \pm 0,23$ & $2,6^{*}$ \\
\hline $\mathrm{Gd}$ & $3,31 \pm 0,42$ & $3,3 \pm 0,5^{*}$ & $15,7 \pm 2,3$ & н.д./n.d. \\
\hline $\mathrm{Tb}$ & $0,39 \pm 0,07$ & $0,45 \pm 0,06$ & $2,27 \pm 0,32$ & н.д./n.d. \\
\hline Dy & $2,80 \pm 0,43$ & $2,7 \pm 0,2$ & $16,2 \pm 1,7$ & н.д./n.d. \\
\hline Ho & $0,55 \pm 0,10$ & $0,6 \pm 0,1$ & $2,90 \pm 0,60$ & н.д./n.d. \\
\hline $\mathrm{Er}$ & $1,51 \pm 0,21$ & $1,4 \pm 0,5$ & $9,78 \pm 1,24$ & н.д./n.d. \\
\hline $\mathrm{Tm}$ & $0,21 \pm 0,07$ & $0,25 \pm 0,02$ & $1,33 \pm 0,19$ & н.д./n.d. \\
\hline $\mathrm{Yb}$ & $1,38 \pm 0,10$ & $1,5 \pm 0,2$ & $10,2 \pm 1,8$ & $7,8^{*}$ \\
\hline $\mathrm{Lu}$ & $0,25 \pm 0,06$ & $0,26 \pm 0,02$ & $1,28 \pm 0,08$ & н.д./n.d. \\
\hline Hf & $3,17 \pm 0,52$ & $3,3 \pm 0,5$ & $10,4 \pm 1,5$ & $13 *$ \\
\hline $\mathrm{Ta}$ & $0,51 \pm 0,13$ & $0,61 \pm 0,04$ & $3,47 \pm 0,49$ & н.д./n.d. \\
\hline $\mathrm{W}$ & н.Д./n.d. & н.д./n.d. & $3,86 \pm 0,41$ & $3,7 *$ \\
\hline $\mathrm{Pb}$ & $22,1 \pm 2,5$ & $22 \pm 4$ & $33,8 \pm 1,2$ & $35 *$ \\
\hline Th & $7,08 \pm 0,50$ & $7 \pm 0,9$ & $45,4 \pm 4,4$ & $45^{*}$ \\
\hline $\mathrm{U}$ & $2,96 \pm 0,36$ & $3,1 \pm 0,4$ & $15,1 \pm 1,0$ & $15^{*}$ \\
\hline
\end{tabular}

Примечание: *-рекомендованные значения; н/д - нет данных.

Note: *-recommended values; n.d. - no data. 
Таблица 3. Сравнение результатов определения элементов в угле и углистом алевролите методом ИСП-МС с использованием разных способов разложения проб (мкг/2)

Table 3. Comparison of the results of ICP-MS determination of elements in coal and carbonaceous siltstone using different methods of sample digestion $(\mu \mathrm{g} / \mathrm{g})$

\begin{tabular}{|c|c|c|c|c|c|c|c|c|}
\hline $\begin{array}{c}\text { Элементы } \\
\text { Еlements }\end{array}$ & 1 & 2 & $\mathrm{~S}_{\mathrm{R}}$ & $\mathrm{RSD}_{\mathrm{R}}$ & 1 & 2 & $\mathrm{~S}_{\mathrm{R}}$ & $\mathrm{RSD}_{\mathrm{R}}$ \\
\hline $\begin{array}{c}\text { Oбразец } \\
\text { Sample }\end{array}$ & \multicolumn{3}{|c|}{$\begin{array}{c}\text { CУ-18-18, уголь } \\
\text { SU-18-18, coal }\end{array}$} \\
\hline $\begin{array}{c}\text { 3ольность \% } \\
\text { Ash соntent, \% }\end{array}$ & \multicolumn{3}{|c|}{5,30} & \multicolumn{3}{|c|}{$\begin{array}{c}\text { CУ-7-18, углистый алевролит } \\
\text { SU-7-18, carbonaceous siltstone }\end{array}$} \\
\hline $\mathrm{Be}$ & 4,99 & 6,55 & 1,10 & 19,12 & 17,59 & 28,67 & 7,83 & 33,87 \\
\hline $\mathrm{Sc}$ & 1,26 & 1,54 & 0,20 & 14,14 & 3,95 & 5,07 & 0,79 & 17,56 \\
\hline $\mathrm{V}$ & 9,77 & 9,64 & 0,09 & 0,95 & 18,09 & 22,48 & 3,10 & 15,30 \\
\hline $\mathrm{Cr}$ & 5,29 & 4,96 & 0,23 & 4,55 & 13,91 & 16,15 & 1,58 & 10,54 \\
\hline $\mathrm{Co}$ & 5,15 & 5,75 & 0,42 & 7,78 & 2,52 & 3,64 & 0,79 & 25,71 \\
\hline $\mathrm{Ni}$ & 1,6 & 1,94 & 0,24 & 13,58 & 3,05 & 11,88 & 6,24 & 83,64 \\
\hline $\mathrm{Cu}$ & 4,82 & 4,25 & 0,40 & 8,89 & 2,76 & 10,19 & 5,25 & 81,14 \\
\hline $\mathrm{Zn}$ & 8,12 & 6,04 & 1,47 & 20,77 & 35,33 & 32,57 & 1,95 & 5,75 \\
\hline $\mathrm{Ga}$ & 1,5 & 1,82 & 0,23 & 13,63 & 27,63 & 32,21 & 3,24 & 10,82 \\
\hline $\mathrm{Rb}$ & 2,98 & 2,90 & 0,06 & 1,92 & 18,96 & 24,84 & 4,16 & 18,99 \\
\hline $\mathrm{Sr}$ & 52,93 & 70,33 & 12,30 & 19,96 & 49,42 & 78,08 & 20,27 & 31,79 \\
\hline $\mathrm{Y}$ & 13,63 & 15,95 & 1,64 & 11,09 & 49,00 & 90,58 & 29,40 & 42,13 \\
\hline $\mathrm{Zr}$ & 10,2 & 14,46 & 3,01 & 24,43 & 68,88 & 161,4 & 65,42 & 56,82 \\
\hline $\mathrm{Nb}$ & 0,69 & 1,08 & 0,28 & 31,16 & 8,98 & 19,04 & 7,11 & 50,77 \\
\hline $\mathrm{Mo}$ & 2,81 & 3,13 & 0,23 & 7,62 & 22,75 & 44,08 & 15,08 & 45,14 \\
\hline $\mathrm{Sn}$ & 0,2 & 0,37 & 0,12 & 42,18 & 2,69 & 6,64 & 2,79 & 59,87 \\
\hline $\mathrm{Ba}$ & 105,1 & 104,2 & 0,66 & 0,64 & 88,01 & 110,9 & 16,19 & 16,27 \\
\hline $\mathrm{Hf}$ & 0,27 & 0,34 & 0,05 & 16,23 & 3,57 & 4,78 & 0,86 & 20,49 \\
\hline $\mathrm{Ta}$ & 0,07 & 0,06 & 0,01 & 10,88 & 1,08 & 1,25 & 0,12 & 10,32 \\
\hline $\mathrm{W}$ & 154,1 & 175,3 & 14,99 & 9,10 & 192,5 & 217,9 & 18,01 & 8,78 \\
\hline $\mathrm{Pb}$ & 2,49 & 2,54 & 0,04 & 1,41 & 10,3 & 14,86 & 3,22 & 25,63 \\
\hline $\mathrm{Th}$ & 2,52 & 1,65 & 0,62 & 29,51 & 26,43 & 22,86 & 2,52 & 10,24 \\
\hline $\mathrm{U}$ & 0,46 & 0,46 & 0,00 & 0,00 & 5,08 & 6,65 & 1,11 & 18,93 \\
\hline
\end{tabular}

Примечание: 1 - открытое кислотное разложение исходных проб; 2 - сплавление золь проб с метаборатом лития. Note: 1 -open acid digestion of the initial samples; 2 - fusion of samples ash with lithium metaborate.

Таблица 4. Сравнение результатов определения содержания элементов методами ИСП-МС и ИНАА (мкг/2)

Table 4. Comparison of the determination results of elements content by ICP-MS and INAA $(\mu \mathrm{g} / \mathrm{g})$

\begin{tabular}{|c|c|c|c|c|c|c|}
\hline $\begin{array}{c}\text { Элементы } \\
\text { Elements }\end{array}$ & $\begin{array}{l}\text { ИСП-МС } \\
\text { ICР-MS }\end{array}$ & $\begin{array}{l}\text { ИНAA } \\
\text { INAA }\end{array}$ & $D_{R}$ & $\begin{array}{l}\text { ИСП-МС } \\
\text { ICР-МS }\end{array}$ & $\begin{array}{l}\text { ИНAA } \\
\text { INAA }\end{array}$ & $D_{R}$ \\
\hline $\begin{array}{c}\text { Образец } \\
\text { Sample }\end{array}$ & \multicolumn{3}{|c|}{$\begin{array}{l}\text { СУ-18-18, уголь } \\
\text { SU-18-18, coal }\end{array}$} & \multicolumn{3}{|c|}{$\begin{array}{l}\text { CУ-7-18, углистый алевролит } \\
\text { SU-7-18, carbonaceous siltstone }\end{array}$} \\
\hline $\mathrm{Sc}$ & 1,26 & 1,40 & 10,23 & 3,95 & 4,41 & 11,00 \\
\hline $\mathrm{Cr}$ & 5,29 & 10,45 & 65,64 & 13,91 & 20,37 & 37,69 \\
\hline Co & 5,15 & 4,96 & 3,73 & 2,52 & 2,83 & 11,59 \\
\hline $\mathrm{Zn}$ & 8,12 & 13,05 & 46,55 & 35,33 & 36,07 & 2,07 \\
\hline As & 15,36 & 13,80 & 10,66 & 103,9 & 112,1 & 7,64 \\
\hline $\mathrm{Rb}$ & 2,98 & 3,37 & 12,20 & 18,96 & 34,31 & 57,63 \\
\hline $\mathrm{Sr}$ & 52,93 & $<40$ & - & 49,42 & $<40$ & - \\
\hline $\mathrm{Sb}$ & 1,42 & 1,08 & 26,97 & 47,36 & 36,00 & 27,26 \\
\hline Cs & 0,84 & 0,74 & 12,40 & 9,31 & 8,33 & 11,11 \\
\hline $\mathrm{Ba}$ & 50,09 & 95,30 & 62,19 & 88,01 & 49,7 & 55,64 \\
\hline $\mathrm{La}$ & 3,93 & 5,07 & 25,22 & 19,93 & 20,91 & 4,80 \\
\hline $\mathrm{Ce}$ & 8,23 & 10,07 & 20,04 & 45,28 & 39,64 & 13,28 \\
\hline $\mathrm{Nd}$ & 4,32 & 2,41 & 56,75 & 12,93 & 5,69 & 77,77 \\
\hline Sm & 1,02 & 1,07 & 5,07 & 3,12 & 3,85 & 20,95 \\
\hline $\mathrm{Eu}$ & 0,21 & 0,17 & 23,27 & 0,34 & 0,38 & 10,06 \\
\hline $\mathrm{Tb}$ & 0,31 & 0,47 & 41,30 & 0,90 & 1,38 & 42,11 \\
\hline $\mathrm{Yb}$ & 1,38 & 1,20 & 13,49 & 6,58 & 5,93 & 10,39 \\
\hline $\mathrm{Lu}$ & 0,20 & 0,18 & 9,67 & 0,90 & 1,12 & 22,13 \\
\hline $\mathrm{Ta}$ & 0,07 & 0,13 & 58,75 & 1,08 & 1,33 & 21,04 \\
\hline Th & 2,52 & 2,28 & 10,24 & 26,43 & 13,20 & 66,77 \\
\hline
\end{tabular}

Сравнительный анализ данных показал допустимое расхождение результатов, полученных методами ИСП-МС и ИНАА. Значения $\mathrm{D}_{\mathrm{R}}$ более 50 \% соответствуют таким элементам, как $\mathrm{Cr}$ (в пробе СУ-18-18), $\mathrm{Rb}$ (в пробе СУ-7-18), Ва и $\mathrm{Nd}$ (в пробах СУ-7-18 и СУ-18-18), Та (в пробе СУ-18-18) и Тh (в пробе СУ-7-18).
Но при содержании элементов менее 100 мкг/г допустимое расхождение между определениями при анализе проб может составлять до 69,9 \% [60, 62]. Определить значение $\mathrm{D}_{\mathrm{R}}$ для $\mathrm{Sr}$ не представилось возможным, так как для этого элемента содержание в пробах находилось на уровне предела обнаружения ИНАА. 


\section{Заключение}

Представленные результаты ИСП-МС анализа трех СО состава золы уноса угля КАТЭКа ГСО 92372008 (ЗУК-2), золы бурого угля Азейского месторождения ГСО 7177-95 (ЗУА-1) и битуминозного угля CLB-1 Геологической службы CША дополняют аттестованные и рекомендованные паспортные значения по содержанию микроэлементов в этих СО. Для образца CLB-1 получены данные по 23 элементам, не указанным в паспорте, таким как $\mathrm{Be}, \mathrm{Ge}, \mathrm{Sr}, \mathrm{Y}, \mathrm{Zr}, \mathrm{Cd}, \mathrm{Sn}$, $\mathrm{Cs}, \mathrm{Pr}, \mathrm{Sm}, \mathrm{Eu}, \mathrm{Gd}, \mathrm{Tb}, \mathrm{Dy}, \mathrm{Ho}, \mathrm{Er}, \mathrm{Tm}, \mathrm{Yb}, \mathrm{Lu}, \mathrm{Hf}, \mathrm{Ta}$, $\mathrm{W}$ и Tl. Образец 3УА-1 охарактеризован по содержанию $\mathrm{Pr}, \mathrm{Nd}, \mathrm{Gd}, \mathrm{Tb}, \mathrm{Dy}, \mathrm{Ho}, \mathrm{Er}, \mathrm{Tm}, \mathrm{Lu}$ и Ta.

В ходе исследований, направленных на выбор оптимальных аналитических условий определения низких содержаний (на уровне $\mathrm{n} \cdot 10^{-2}$ мкг/г) широкого набора химических элементов в углях (угольных материалах), рассмотрена возможность применения отдельных методик с использованием высокочувствительных инструментальных методов - ИСП-МС и ИНАА.

Проведенное сопоставление ИСП-МС результатов анализа для проб углей и углистых пород после различных способов подготовки проб: методики открытого кислотного разложения и сплавления с метаборатом лития, показало особенности поведения некоторых элементов в процессе обозначенных способов химической пробоподготовки. Отмечена возможность получения «заниженных» результатов при определении $\mathrm{Zr}, \mathrm{Hf}, \mathrm{Nb}, \mathrm{Ta}$ и $\mathrm{Sn}$ с использованием методики открытого кислотного разложения. Исходя из этого,

\section{СПИСОК ЛИТЕРАТУРЫ}

1. Юдович Я.Э., Кетрис М.П. Токсичные элементы-примеси в ископаемых углях. - Екатеринбург: Изд-во УрО РАН, 2005. $648 \mathrm{c}$.

2. Dai S., Graham I.T., Ward C.R. A review of anomalous rare earth elements and yttrium in coal // International Journal of Coal Geology. - 2016. - V. 159. - P. 82-95.

3. Dai S., Finkelman R.B. Coal as a promising source of critical elements: progress and future prospects // International Journal of Coal Geology. - 2018. - V. 186. - P. 155-164.

4. Recovery of metals and other beneficial products from coal fly ash: a sustainable approach for fly ash management / P.K. Sahoo, K. Kim, M.A. Powell, S.M. Equeenuddin // International Journal of Coal Science \& Technology. - 2016. - V. 3. - P. 267-283.

5. A comparative study on the mineralogy, chemical speciation, and combustion behavior of toxic elements of coal beneficiation products / B. Fu, G. Liu, M. Sun, J.C. Hower, G. Hu, D. Wu // Fuel. 2018. - V. 228. - P. 297-308.

6. Finkelman R.B. Potential health impact of burning coal beds and waste bank. Coal fires burning around the World: a global catastrophe // International Journal of Coal Geology. - 2004. - V. 59. № 1-2. - P. 9-24.

7. ГОСТ 33130-2014. Угли бурые, каменные и антрацит. Номенклатура показателей качества (Переиздание). - М.: Стандартинформ, 2019. - 14 с

8. Арбузов С.И., Ершов В.В. Геохимия редких элементов в углях Сибири. - Томск: Д-Принт, 2007 - 468 с.

9. Finkelman R.B., Palmer C.A., Wang P. Quantification of the modes of occurrence of 42 elements in coal // International Journal of Coal Geology. - 2018. - V. 185. - P. 138-160.

10. Stuckman M.Y., Lopano C.L., Granite E.J. Distribution and speciation of rare earth elements in coal combustion by-products via synchrotron microscopy and spectroscopy // International Journal of Coal Geology. - 2018. - V. 195. - P. 125-138. определены ограничения по применению рассмотренных методических подходов для анализа различных по составу (зольности) проб углей и углистых пород методом ИСП-МС. Для указанных элементов полнота вскрытия проб с зольностью выше 30 \% достигается только после сплавления зольного материала с метаборатом лития, а анализ подразумевает пересчет получаемых данных на исходную пробу, с учетом ее зольности. В то же время была выявлена возможность «завышения» результатов при определении методом ИСП-МС таких элементов, как $\mathrm{Cu}$ и $\mathrm{Ni}$, в углях и углистых породах по этой методике пробоподготовки. Исчерпывающего объяснения данного факта в текущий момент найдено не было. В целом к нашей рекомендации для точного определения $\mathrm{Cu}$ и $\mathrm{Ni}$ можно отнести использование методики кислотного разложения проб, даже при их высокой (более 30 \%) зольности.

На основании метрологической оценки и сопоставления экспериментальных данных, полученных в двух независимых лабораториях, показана перспективность использования методов ИСП-МС и ИНАА для количественного определения содержания микроэлементов в углях и углистых породах с требуемой при геохимических исследованиях точностью. При этом для таких элементов, как $\mathrm{Cr}, \mathrm{Rb}, \mathrm{Ba}, \mathrm{Nd}, \mathrm{Ta}$ и Th, отмечено достаточно высокое $\left(\mathrm{D}_{\mathrm{R}} 50 \%\right)$ относительное расхождение результатов между ИСП-МС и ИНАА.

Исследование выполнено при финансовой поддержке Российского научного фонда в рамках проекта № 18-17-00004.

11. Distribution of rare earth elements in coal combustion fly ash, determined by SHRIMP-RG ion microprobe / A. Kolker, C. Scott, J.C. Hower, J.A. Vazquez, C.L. Lopano, S. Dai // International Journal of Coal Geology. - 2017. - V. 184. - P. 1-10.

12. Determination of lead in coal using direct solid sampling and highresolution continuum source graphite furnace atomic absorption spectrometry / D.L.G. Borges, A.F. Silva, A.J. Curtius, B. Welz, U. Heitmann // Microchimica Acta. - 2006. - V. 154. - № 1-2. P. 101-107.

13. Borges D.L.G., Welz B., Curtius A.J. Determination of As, Cd, Pb and $\mathrm{Tl}$ in coal by electrothermal vaporization unductively coupled plasma mass spectrometry using slurry sampling and external calibration against aqueous standards // Microchimica Acta. - 2007. V. 159. - № 1-2. - P. 19-26.

14. Development of an accurate, sensitive, and robust isotope dilution laser ablation ICP-MS method for simultaneous multi-element analysis (chlorine, sulfur, and heavy metals) in coal samples / S.F. Boulyga, J. Heilmann, T. Prohaska, K.G. Heumann // Analytical and Bioanalytical Chemistry. - 2007. - V. 389. - № 3. P. 697-706.

15. Rodushkin I., Axelsson M.D., Burman E. Multielement analysis of coal by ICP techniques using solution nebulization and laser ablation // Talanta. - 2000. - V. 51. - № 4. - P. 743-759.

16. Major, trace, and natural radioactive elements in bituminous coal from Australia, Romania, Russia, South Africa and Ukraine: a comparative study / O.G. Duliu, O.A. Culicov, I. Rãdulescu, C. Cristea, T. Vasiu // Journal of Radioanalytical and Nuclear Chemistry. - 2005. - V. 264. - № 3. - P. 525-534.

17. Comments on the geochemistry of rare-earth elements ( $\mathrm{La}, \mathrm{Ce}, \mathrm{Sm}$, $\mathrm{Eu}, \mathrm{Tb}, \mathrm{Yb}, \mathrm{Lu}$ ) with examples from coals of north Asia (Siberia, Russian Far East, North China, Mongolia, and Kazakhstan) / S.I. Arbuzov, I.Y. Chekryzhov, R.B. Finkelman, Y.Z. Sun, C.L. Zhao, S.S. Il'enok, M.G. Blokhin, N.V. Zarubina // International Journal of Coal Geology. - 2019. - V. 206. - P. 106-120. 
18. Особенности анализа микроэлементов в бурых углях методом масс-спектрометрии с индуктивно связанной плазмой / Г.А. Олейникова, В.Л. Кудряшов, В.И. Вялов, Я.Ю. Фадин // Химия твердого топлива. - 2015. - № 2. - С. 51-58.

19. Determination of Eu concentrations in coal, fly ash and sedimentary rocks using a cation exchange resin and inductively coupled plasma mass spectrometry (ICP-MS) / X. Yan, S. Dai, I.T. Graham, X. He, K. Shan, X. Liu // International Journal of Coal Geology. 2018. - V. 191. - P. 152-156.

20. Nadkarni R.A. Multitechnique multielemental analysis of coal and fly ash // Analytical Chemistry. - 1980 - V. 52. - № 6. - P. 929-935.

21. Single-step microwave digestion with $\mathrm{HNO}_{3}$ alone for determination of trace elements in coal by ICP spectrometry / J. Wang, K. Sakanishi, O. Yamada, H. Tao, I. Saito // Talanta. - 2006. V. 68. - P. 1584-1590.

22. Fluoride and chloride determination in fossil fuels after sample preparation by pyrohydrolysis / F.G. Antes, F.A. Duarte, E.L.M. Flores, J.N.G. Paniz, E.M.M. Flores, V.L. Dressler // Quimica Nova. - 2010. - V. 33. - № 5. - P. 1130-1134.

23. Potentiometric determination of fluoride in geological and biological samples following pyrohydrolytic decomposition / V.L. Dressler, D. Pozebon, E.L.M. Flores, J.N.G. Paniz, E.M.M. Flores // Analytica Chimica Acta. - 2002. - V. 466. - № 1. - P. 117-123.

24. Utilization of oxygen flask combustion method for the determination of mercury and sulfur in coal / W. Geng, T. Nakajima, H. Takanishi, A. Ohki // Fuel. - 2008. - V. 87. - № 4-5. - P. 559-564.

25. Determination of halogens in coal after digestion using the microwave-induced combustion technique / E.M.M. Flores, M.F. Mesko, D.P. Moraes, J.S.F. Pereira, P.A. Mello, J.S. Barin, G. Knapp // Analytical Chemistry. - 2008. - V. 80 - № 6. - P. 1865-1870.

26. Sample preparation techniques based on combustion reactions in closed vessels - a brief overview and recent applications E.M.M. Flores, J.S. Barin, M.F. Mesko, G. Knapp // Spectrochimica Acta Part B. - 2007. - V. 62. - № 9. - P. 1051-1064.

27. Yuan C.G. Leaching characteristics of metals in fly ash from coalfired power plant by sequential extraction procedure // Microchimica Acta. - 2009. - V. 165. - № 1-2. - P. 91-96.

28. Spears D.A. The determination of trace element distributions in coals using sequential chemical leaching - a new approach to an old method // Fuel. - 2013. - V. 114. - P. 31-37.

29. ASTM D 6357-19: Standard test methods for determination of trace elements in coal, coke, and combustion residues from coal utilization processes by inductively coupled plasma atomic emission spectrometry, inductively coupled plasma mass spectrometry, and graphite furnace atomic absorption spectrometry. - West Conshohocken, PA: ASTM International, 2019. URL: http://www.astm.org/cgi-bin/resolver.cgi?D6357-19 (дата обращения 14.10.2020)

30. ASTM D 6349-13: Standard test method for determination of major and minor elements in coal, coke, and solid residues from combustion of coal and coke by inductively coupled plasma atomic-emission spectrometry. - West Conshohocken, PA: ASTM International, 2013. URL: http://www.astm.org/cgi-bin/resolver.cgi? D6349-13 (дата обращения 14.10.2020).

31. ASTM D 3683-11: Standard test method for trace elements in coal and coke ash by atomic absorption (withdrawn 2018). - West Conshohocken, PA: ASTM International, 2011. URL: http://www.astm.org/ cgi-bin/resolver.cgi?D3683-11 (дата обращения 14.10.2020).

32. ГОСТ Р 54237-2010. Топливо твердое минеральное. Определение химического состава золы методом атомноэмиссионной спектрометрии с индуктивно связанной плазмой. - М.: Стандартинформ, 2012. - 12 с.

33. Jain J.C., Neal C.R., Hanchar J.M. Problems associated with the determination of rare earth elements of a «gem» quality zircon by inductively coupled plasma-mass spectrometry // Geostandards and Geoanalytical Research. - 2001. - V. 25. № 2-3. - P. 299-237.

34. New REE and trace elements data in two kimberlitic reference materials by ICP-MS / P. Roy, V. Balaram, A. Kumar, M. Satyanarayanan, G.T. Rao // Geostandards and Geoanalytical Research. - 2007. - V. 31. - № 3. - P. 261-273.

35. Yokoyama T., Makishima A., Nakamura E. Evaluation of the coprecipitation of incompatible trace elements with fluoride during silicate rock dissolution by acid digestion // Chemical Geology. 1999. - V. 157. - № 3-4. - P. 175-187.
36. Wilson M.A., Burt R., Lee C.W. Improved elemental recoveries in soils with heating boric acid following microwave total digestion // Communications in Soil Science and Plant Analysis. - 2006. V. 37. - № 3-4. - P. 513-524.

37. Yu Z., Robinson P., McGoldrick P. An evaluation of methods for the chemical decomposition of geological material for trace elements determination using ICP-MS // Geostandards and Geoanalytical Research. - 2001. - V. 25. - № 2-3. - P. 199-217.

38. Liang Q., Jing H., Gregoire D.C. Determination of trace elements in granites by inductively coupled plasma-mass spectrometry // Talanta. - 2000. - V. 51. - № 3. - P. 507-513.

39. Trends in sample preparation using combustion techniques / J.S. Barin, E.M.M. Flores, G. Knapp, M.A.Z. Arruda // Trends in sample preparation. - New York: Nova Science Publ., 2006.P. 73-114.

40. Horvat M., Lupsina V., Pihlar B. Determination of total mercury in coal fly ash by gold amalgamation cold vapour atomic absorption spectrometry // Analytica Chimica Acta. - 1991. - V. 243. P. 71-79.

41. Dias.M.D., Satte M.S.S. Determination of As in environmental organic samples by atomic absorption spectrometry with electrothermal atomization after $\mathrm{O}_{2}$ bomb combustion // Quimica Nova. 2003. - V. 26. - № 5. - P. 661-664.

42. Mester Z., Sturgeon R. Sample preparation for trace element analysis // Comprehensive analytical chemistry. Volume XLI. - Amsterdam: Elsevier, 2003. - P. 201-203.

43. Srogi K. Microwave-assisted sample preparation of coal and coal fly ash for subsequent metal determination // Analytical Letters. 2007. - V. 40. - № 2. - P. 199-232.

44. Mketo N., Nomngongo P.N., Ngila J.C. A rapid microwaveassisted acid extraction method based on the use of diluted $\mathrm{HNO}_{3}$ $\mathrm{H}_{2} \mathrm{O}_{2}$ followed by ICP-MS analysis for simultaneous determination of trace elements in coal samples // International Journal of Environmental Analytical Chemistry. - 2015. - V. 95. № 5. - P. 453-465.

45. Wang C.Y., H.F. Ding, Yang D.D. Determination of uranium in coal fly ash by inductively coupled plasma mass spectrometry with microwave digestion // Yejin Fenxi. - 2019. - V. 39. - № 6. P. 20-23.

46. An integrated approach based on oxidative pyrolysis and microwave-assisted digestion for the multi-elemental analysis of coal samples by ICP-based techniques / K.M.V. Balarama, K. Chandrasekaran, S. Chakravarthy, D. Karunasagar // Fuel. 2015. - V. 158. - P. 770-778.

47. Determination of metals and metalloids in light and heavy crude oil by ICP-MS after digestion by microwave-induced combustion / J.S.F. Pereira, D.P. Moraes, F.G. Antes, L.O. Diehl, M.F.P. Santos, R.C.L. Guimarães, T.C.F. Fonseca, V.L. Dressler, E.M.M. Flores // Microchemical Journal. - 2010. - V. 96. - № 1. - P. 4-11.

48. Evaluation of sample preparation methods for elastomer digestion for further halogens determination / D.P. Moraes, J.S.F. Pereira, L.O. Diehl, M.F. Mesko, V.L. Dressler, J.N.G. Paniz, G. Knapp, E.M.M. Flores // Analytical and Bioanalytical Chemistry. 2010. - V. 397. - № 2. - P. 563-570.

49. Feasibility of microwave-induced combustion for digestion of crude oil vacuum distillation residue for chlorine determination / J.S.F. Pereira, P.A. Mello, F.A. Duarte, M.F.P. Santos, G. Knapp, V.L. Dressler, E.M.M. Flores // Energy Fuels. - 2009. - V. 23. № 12. - P. 6015-6019.

50. Seafood digestion by microwave-induced combustion for total arsenic determination by atomic spectrometry techniques with hydride generation / F.A. Duarte, J.S.F. Pereira, J.S. Barin, M.F. Mesko, V.L. Dressler, E.M.M. Flores, G. Knapp // Journal of Analytical Atomic Spectrometry. - 2009. - V. 24. - № 2.P. 224-227.

51. Determination of toxic elements in coal by ICP-MS after digestion using microwave-induced combustion / F.G. Antes, F.A. Duarte, M.F. Mesko, M.A.G. Nunes, V.A. Pereira, E.I. Müller, V.L. Dressler, E.M.M. Flores // Talanta. - 2010. - V. 83. - № 2. P. 364-369.

52. Сравнительная оценка содержания рения в углеродсодержащих горных породах методами масс-спектрометрии с индуктивно связанной плазмой и инструментального нейтронноактивационного анализа / В.В. Иванов, Н.В. Зарубина, В.Г. Зиновьев, И.С. Окунев, Г.И. Шуляк, Д.С. Остапенко // Вопросы 
атомной науки и техники. Серия: Ядерно-реакторные константы. - 2015. - Спецвыпуск. - C. 49-58.

53. Ostapenko D.S., Zarubuna N.V., Ivanov V.V. Determination of rhenium in carbon-terigenous, organo-mineral and organogenic geological samples by ICP-MS method $/ / 10^{\text {th }}$ International Symposium on Technetium and Rhenium - Science and Utilization. Moscow: Publishing House Granica, 2018. - P. 45-46.

54. Оценка рениеносности углей методом масс-спектрометрии с индуктивно связанной плазмой / Н.В. Зарубина, В.В. Иванов, Д.С. Остапенко, М.Г. Блохин, Е.Г. Панова, И.С. Окунев // Комплексное использование потенциала каменных и бурых углей и создание комбинированных экологически безопасных технологий их освоения: Всерос. конф. с междунар. участием: сб. докладов. - Благовещенск: АмурНЦ ДВО РАН, ИГиП ДВО РАН, 2017. - С. 133-137.

55. Distribution of rare earth and selected trace elements in combustion products of Yerkovetskoe brown coal deposit (Amur Region, Russia) / A.P. Sorokin, A.A. Konyushok, O.A. Ageev, N.V. Zarubina, V.V. Ivanov, J. Wang // Energy Exploration \& Exploitation. - 2019. - V. 37. - № 6. - P. 1721-1736.

56. Середин В.В. Металлоносность углей: условия формирования и перспективы освоения // Угольная база России. Т. VI. - М.: Геоинформмарк, 2004. - С. 453-519.

57. Седых А.К. Кайнозойские рифтогенные впадины Приморья (геологическое строение, минерагения и геодинамика углегенеза). - Владивосток: Дальнаука, 2008. - 248 с.
58. Судыко А.Ф. Определение урана, тория, скандия и некоторых редкоземельных элементов в двадцати четырех стандартных образцах сравнения инструментальным нейтронноактивационным методом // Радиоактивность и радиоактивные элементы в среде обитания человека: Материалы V Межд. конф. - Томск: STT, 2016. - С. 620-624.

59. Дворкин В.И. Метрология и обеспечение качества химического анализа. - М.: Техносфера, 2019. - 318 с

60. ОСТ 41-08-212-04. Управление качеством аналитических работ. Нормы погрешности при определении химического состава минерального сырья и классификация методик лабораторного анализа по точности результатов. - М.: ФГУП ВИМС, 2004. -23 c.

61. Оценка качества элементного анализа силикатных горных пород по результатам участия в международной программе профессионального тестирования геоаналитических лабораторий - GEOPT / А.И. Кузнецова, Л.Л. Петров, А.И. Финкельштейн, В.И. Меньшиков // Аналитика и контроль. - 2002. - Т. 6. - № 5. - С. 584-592.

62. ОСТ 41-08-272-04. Управление качеством аналитических работ. Методы геологического контроля качества аналитических работ. - М.: ФГУП ВИМС, 2004. - 43 с.

Поступила 24.02.2021 2.

\section{Информация об авторах}

Зарубина Н.B., научный сотрудник лаборатории аналитической химии Дальневосточного геологического института ДВО РАН

Блохин М.Г., кандидат биологических наук, заместитель директора по научной работе Дальневосточного геологического института ДВО РАН.

Oстапенко Д.С., младший научный сотрудник лаборатории аналитической химии Дальневосточного геологического института ДВО РАН.

Чекрыжов И.Ю., научный сотрудник лаборатории геохимии Дальневосточного геологического института ДВО РАН.

Арбузов С.И., доктор геолого-минералогических наук, профессор отделения геологии Инженерной школы природных ресурсов Национального исследовательского Томского политехнического университета.

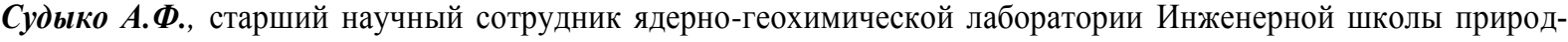
ных ресурсов Национального исследовательского Томского политехнического университета. 
UDC 543.51:543.53:553.96

\title{
ANALYTICAL APPROACHES TO THE QUANTITATIVE DETERMINATION OF THE CHEMICAL ELEMENTS CONTENT IN COALS AND COAL ROCKS USING ICP-MS AND INAA METHODS
}

\author{
Natalya V. Zarubina ${ }^{1}$, \\ zarubina@fegi.ru
}

\author{
Maksim G. Blokhin ${ }^{1}$, \\ blokhin@fegi.ru
}

Dmitry S. Ostapenko1, insunday888@gmail.com

\author{
Igor Yu. Chekryzhov ${ }^{1}$, \\ chekr2004@mail.ru
}

Sergey I. Arbuzov², siarbuzov@mail.ru

\author{
Alexander F. Sudyko², \\ sudykoAF@yandex.ru \\ 1 Far East Geological Institute FEB RAS, \\ 159, 100-letie Vladivostoka avenue, Vladivostok, 690022, Russia. \\ 2 National Research Tomsk Polytechnic University, \\ 30, Lenin avenue, Tomsk, 634050, Russia.
}

The relevance of the study is caused by significant interest of scientists working in the field of coal geology and specialists of chemical and analytical laboratories practicing quantitative elemental analysis of environmental objects, to the problem of high-precision determination of low concentrations of chemical elements in coals and coal materials for the subsequent use of the obtained data in solving scientific and various technological tasks.

The main aim is to consider and generalize the most frequently used methodological approaches to the determination of chemical elements in a wide range of their contents in coals (coal materials), as well as to assess the precision and accuracy of the ICP-MS method results obtained after open acid decomposition of samples and their fusion with lithium metaborate, comparing data with the INAA results method and statistical data processing.

Objects: coals, carbonaceous siltstones and coalified timber from the Spetsugli germanium deposit, located on the southwestern edge of the Khanka massif in the eastern part of the Pavlovskoe coal-bearing depression, Primorsky Krai.

Methods: inductively coupled plasma mass spectrometry (ICP-MS), applied after transferring the studied samples to a dissolved form, and a non-destructive quantitative method of instrumental neutron activation analysis (INAA). Methods of mathematical statistics were used to process the obtained results.

Results. The results of ICP-MS analysis of three certified reference materials (CRM) of fly ash from KATEK GSO 9237-2008 (ZUK-2), brown coal ash from the Azeiskoe coal deposit GSO 7177-95 (ZUA-1) and bituminous CLB-1 coal of the US Geological Survey were obtained, presented in the form of data on their trace element composition in comparison with the certified and recommended values. The results of the determination of some elements given in the paper supplement the data on these CRMs presented in the certificate. Comparison of ICP-MS analysis results for samples of coals and carbonaceous rocks after different methods of chemical sample preparation: technique of open acid digestion and fusion with lithium metaborate, was carried out. The behavior of individual elements during these processes has been studied. Matching the obtained data by ICP-MS and INAA methods is performed. All results were processed metrologically and their permissible discrepancy was shown.

\section{Key words:}

Inductively coupled plasma mass spectrometry, instrumental neutron activation analysis, certified reference materials, chemical sample preparation, coal, carbonaceous rocks, Spetsugli, Pavlovsk coal-bearing depression.

The research was carried out with the financial support of the Russian Science Foundation within the framework of project No. 18-17-00004.

\section{REFERENCES}

1. Yudovich Ya.E., Ketris M.P. Toksichnye elementy-primesi $v$ iskopaemykh uglyakh [Toxic trace elements in fossil coals]. Ekaterinburg, UrO RAN Publ., 2005. 648 p.

2. Dai S., Graham I.T., Ward C.R. A review of anomalous rare earth elements and yttrium in coal. International Journal of Coal Geology, 2016, vol. 159, pp. 82-95.

3. Dai S., Finkelman R.B. Coal as a promising source of critical elements: progress and future prospects. International Journal of Coal Geology, 2018, vol. 186, pp. 155-164.
4. Sahoo P.K., Kim K., Powell M.A., Equeenuddin S.M. Recovery of metals and other beneficial products from coal fly ash: a sustainable approach for fly ash management. International Journal of Coal Science \& Technology, 2016, vol. 3, pp. 267-283.

5. Fu B., Liu G., Sun M., Hower J.C., Hu G., Wu D. A comparative study on the mineralogy, chemical speciation, and combustion behavior of toxic elements of coal beneficiation products. Fuel, 2018, vol. 228, pp. 297-308.

6. Finkelman R.B. Potential health impact of burning coal beds and waste bank. Coal fires burning around the World: a global catas- 
trophe. International Journal of Coal Geology, 2004, vol. 59, no. $1-2$, pp. 9-24.

7. GOST 33130-2014. Ugli burye, kamennye i antratsit. Nomenklatura pokazateley kachestva (Pereizdanie) [State Standard 331302014. Brown coals, stone and anthracite. Quality score nomenclature (Reissue)]. Moscow, StandartInform Publ., 2019. 14 p.

8. Arbuzov S.I., Ershov V.V. Geokhimiya redkikh elementov v ug lyakh Sibiri [Geochemistry of trace elements in Siberian coals]. Tomsk, D-Print Publ., 2007. 468 p.

9. Finkelman R.B., Palmer C.A., Wang P. Quantification of the modes of occurrence of 42 elements in coal. International Journal of Coal Geology, 2018, vol. 185, pp. 138-160.

10. Stuckman M.Y., Lopano C.L., Granite E.J. Distribution and speciation of rare earth elements in coal combustion by-products via synchrotron microscopy and spectroscopy. International Journal of Coal Geology, 2018, vol. 195, pp. 125-138.

11. Kolker A., Scott C., Hower J.C., Vazquez J.A., Lopano C.L., Dai S. Distribution of rare earth elements in coal combustion fly ash, determined by SHRIMP-RG ion microprobe. International Journal of Coal Geology, 2017, vol. 184, pp. 1-10.

12. Borges D.L.G., Silva A.F., Curtius A.J., Welz B., Heitmann U. Determination of lead in coal using direct solid sampling and highresolution continuum source graphite furnace atomic absorption spectrometry. Microchimica Acta, 2006, vol. 154, no. 1-2, pp. 101-107.

13. Borges D.L.G., Welz B., Curtius A.J. Determination of $\mathrm{As}, \mathrm{Cd}, \mathrm{Pb}$ and $\mathrm{Tl}$ in coal by electrothermal vaporization unductively coupled plasma mass spectrometry using slurry sampling and external calibration against aqueous standards. Microchimica Acta, 2007, vol. 159 , no. 1-2, pp. 19-26.

14. Boulyga S.F., Heilmann J., Prohaska T., Heumann K.G. Development of an accurate, sensitive, and robust isotope dilution laser ablation ICP-MS method for simultaneous multi-element analysis (chlorine, sulfur, and heavy metals) in coal samples. Analytical and Bioanalytical Chemistry, 2007, vol. 389, no. 3, pp. 697-706.

15. Rodushkin I., Axelsson M.D., Burman E. Multielement analysis of coal by ICP techniques using solution nebulization and laser ablation. Talanta, 2000, vol. 51, no. 4, pp. 743-759.

16. Duliu O.G., Culicov O.A., Rãdulescu I., Cristea C., Vasiu T. Major, trace, and natural radioactive elements in bituminous coal from Australia, Romania, Russia, South Africa and Ukraine: a comparative study. Journal of Radioanalytical and Nuclear Chemistry, 2005, vol. 264, no. 3, pp. 525-534.

17. Arbuzov S.I., Chekryzhov I.Y., Finkelman R.B., Sun Y.Z., Zhao C.L., Il'enok S.S., Blokhin M.G., Zarubina N.V. Comments on the geochemistry of rare-earth elements $(\mathrm{La}, \mathrm{Ce}, \mathrm{Sm}, \mathrm{Eu}, \mathrm{Tb}$, $\mathrm{Yb}, \mathrm{Lu}$ ) with examples from coals of north Asia (Siberia, Russian Far East, North China, Mongolia, and Kazakhstan). International Journal of Coal Geology, 2019, vol. 206, pp. 106-120.

18. Oleinikova G.A., Kudryashov V.L., Vyalov V.I., Fadin Y.Y. Determination of trace elements in brown coals by inductively coupled plasma mass spectrometry. Solid Fuel Chemistry, 2015, vol. 49, pp. 109-116.

19. Yan X., Dai S., Graham I.T., He X., Shan K., Liu X. Determination of Eu concentrations in coal, fly ash and sedimentary rocks using a cation exchange resin and inductively coupled plasma mass spectrometry (ICP-MS). International Journal of Coal Geology, 2018, vol. 191, pp. 152-156.

20. Nadkarni R.A. Multitechnique multielemental analysis of coal and fly ash. Analytical Chemistry, 1980, vol. 52, no. 6, pp. 929-935.

21. Wang J., Sakanishi K., Yamada O., Tao H., Saito I. Single-step microwave digestion with $\mathrm{HNO}_{3}$ alone for determination of trace elements in coal by ICP spectrometry. Talanta, 2006, vol. 68, pp. 1584-1590.

22. Antes F.G., Duarte F.A., Flores E.L.M., Paniz J.N.G., Flores E.M.M., Dressler V.L. Fluoride and chloride determination in fossil fuels after sample preparation by pyrohydrolysis. Quimica Nova, 2010, vol. 33, no. 5, pp. 1130-1134.

23. Dressler V.L., Pozebon D., Flores E.L.M., Paniz J.N.G., Flores E.M.M. Potentiometric determination of fluoride in geological and biological samples following pyrohydrolytic decomposition. Analytica Chimica Acta, 2002, vol. 466, no. 1, pp. 117-123.

24. Geng W., Nakajima T., Takanishi H., Ohki A. Utilization of oxygen flask combustion method for the determination of mercury and sulfur in coal. Fuel, 2008, vol. 87, no. 4-5, pp. 559-564.
25. Flores E.M.M., Mesko M.F., Moraes D.P., Pereira J.S.F., Mello P.A., Barin J.S., Knapp G. Determination of halogens in coal after digestion using the microwave-induced combustion technique. Analytical Chemistry, 2008, vol. 80, no. 6, pp. 1865-1870.

26. Flores E.M.M., Barin J.S., Mesko M.F., Knapp G. Sample preparation techniques based on combustion reactions in closed vessels - a brief overview and recent applications. Spectrochimica Acta Part B, 2007, vol. 62, no. 9, pp. 1051-1064.

27. Yuan C.G. Leaching characteristics of metals in fly ash from coalfired power plant by sequential extraction procedure. Microchimica Acta, 2009, vol. 165, no. 1-2, pp. 91-96.

28. Spears D.A. The determination of trace element distributions in coals using sequential chemical leaching - a new approach to an old method. Fuel, 2013, vol. 114, pp. 31-37.

29. ASTM D 6357-19: Standard test methods for determination of trace elements in coal, coke, and combustion residues from coal utilization processes by inductively coupled plasma atomic emission spectrometry, inductively coupled plasma mass spectrometry, and graphite furnace atomic absorption spectrometry. West Conshohocken, PA, ASTM International, 2019. Available at: http://www.astm.org/cgi-bin/resolver.cgi?D6357-19 (accessed 14 October 2020).

30. ASTM D 6349-13: Standard test method for determination of major and minor elements in coal, coke, and solid residues from combustion of coal and coke by inductively coupled plasma atomic-emission spectrometry. West Conshohocken, PA, ASTM International, 2013. Available at: http://www.astm.org/cgi-bin/resolver. cgi?D6349-13 (accessed 14 October 2020).

31. ASTM D 3683-11: Standard test method for trace elements in coal and coke ash by atomic absorption (withdrawn 2018). West Conshohocken, PA, ASTM International, 2011. Available at: http://www.astm.org/cgi-bin/resolver.cgi?D3683-11 (accessed 14 October 2020).

32. GOST R 54237-2010. Toplivo tverdoe mineralnoe. Opredelenie khimicheskogo sostava zoly metodom atomno-emissionnoy spektrometrii s induktivno svyazannoy plazmoy [State Standard R 54237-2010. Solid mineral fuel. Determination of the chemical composition of ash by inductively coupled plasma atomic emission spectrometry]. Moscow, StandartInform Publ., 2012. 12 p.

33. Jain J.C. Neal C.R., Hanchar J.M. Problems associated with the determination of rare earth elements of a «gem» quality zircon by inductively coupled plasma-mass spectrometry. Geostandards and Geoanalytical Research, 2001, vol. 25, no. 2-3, pp. 299-237.

34. Roy P., Balaram V., Kumar A., Satyanarayanan M., Rao G.T. New REE and trace elements data in two kimberlitic reference materials by ICP-MS. Geostandards and Geoanalytical Research, 2007, vol. 31, no. 3, pp. 261-273.

35. Yokoyama T., Makishima A., Nakamura E. Evaluation of the coprecipitation of incompatible trace elements with fluoride during silicate rock dissolution by acid digestion. Chemical Geology, 1999, vol. 157, no. 3-4, pp. 175-187.

36. Wilson M.A., Burt R., Lee C.W. Improved elemental recoveries in soils with heating boric acid following microwave total digestion. Communications in Soil Science and Plant Analysis, 2006, vol. 37, no. 3-4, pp. 513-524.

37. Yu Z., Robinson P., McGoldrick P. An evaluation of methods for the chemical decomposition of geological material for trace elements determination using ICP-MS. Geostandards and Geoanalytical Research, 2001, vol. 25, no. 2-3, pp. 199-217.

38. Liang Q., Jing H., Gregoire D.C. Determination of trace elements in granites by inductively coupled plasma-mass spectrometry. $\mathrm{Ta}$ lanta, 2000, vol. 51, no. 3, pp. 507-513.

39. Barin J.S., Flores E.M.M., Knapp G., Arruda M.A.Z. Trends in sample preparation using combustion techniques. Trends in sample preparation. New York, Nova Science Publ., 2006. pp. 73-114.

40. Horvat M., Lupsina V., Pihlar B. Determination of total mercury in coal fly ash by gold amalgamation cold vapour atomic absorption spectrometry. Analytica Chimica Acta, 1991, vol. 243, pp. 71-79.

41. Dias V.M.D., Satte M.S.S. Determination of As in environmental organic samples by atomic absorption spectrometry with electrothermal atomization after $\mathrm{O}_{2}$ bomb combustion. Quimica Nova, 2003, vol. 26, no. 5, pp. 661-664

42. Mester Z., Sturgeon R. Sample preparation for trace element analysis. Comprehensive analytical chemistry. Volume XLI. Amsterdam, Elsevier Publ., 2003. pp. 201-203. 
43. Srogi K. Microwave-assisted sample preparation of coal and coal fly ash for subsequent metal determination. Analytical Letters, 2007, vol. 40, no. 2, pp. 199-232.

44. Mketo N., Nomngongo P.N., Ngila J.C. A rapid microwave-assisted acid extraction method based on the use of diluted $\mathrm{HNO}_{3}-\mathrm{H}_{2} \mathrm{O}_{2}$ followed by ICP-MS analysis for simultaneous determination of trace elements in coal samples. International Journal of Environmental Analytical Chemistry,2015, vol. 95, no. 5, pp. 453-465.

45. Wang C.Y., Ding H.F., Yang D.D. Determination of uranium in coal fly ash by inductively coupled plasma mass spectrometry with microwave digestion. Yejin Fenxi, 2019, vol. 39, no. 6, pp. 20-23.

46. Balarama K.M.V., Chandrasekaran K., Chakravarthy S., Karunasagar D. An integrated approach based on oxidative pyrolysis and microwave-assisted digestion for the multi-elemental analysis of coal samples by ICP-based techniques. Fuel, 2015, vol. 158 , pp. $770-778$.

47. Pereira J.S.F., Moraes D.P., Antes F.G., Diehl L.O., Santos M.F.P., Guimarães R.C.L., Fonseca T.C.F., Dressler V.L., Flores Determination of metals and metalloids in light and heavy crude oil by ICP-MS after digestion by microwave-induced combustion. $M i$ crochemical Journal, 2010, vol. 96, no. 1, pp. 4-11.

48. Moraes D.P., Pereira J.S.F., Diehl L.O., Mesko M.F., Dressler V.L., Paniz J.N.G., Knapp G., Flores E.M.M. Evaluation of sample preparation methods for elastomer digestion for further halogens determination. Analytical and Bioanalytical Chemistry, 2010, vol. 397, no. 2, pp. 563-570.

49. Pereira J.S.F., Mello P.A., Duarte F.A., Santos M.F.P., Knapp G., Dressler V.L., Flores E.M.M. Feasibility of microwave-induced combustion for digestion of crude oil vacuum distillation residue for chlorine determination. Energy Fuels, 2009, vol. 23, no. 12, pp. 6015-6019.

50. Duarte F.A., Pereira J.S.F., Barin J.S., Mesko M.F., Dressler V.L., Flores E.M.M., Knapp G. Seafood digestion by microwaveinduced combustion for total arsenic determination by atomic spectrometry techniques with hydride generation. Journal of Analytical Atomic Spectrometry, 2009, vol. 24, no. 2, pp. 224-227.

51. Antes F.G., Duarte F.A., Mesko M.F., Nunes M.A.G., Pereira V.A., Müller E.I., Dressler V.L., Flores E.M.M. Determination of toxic elements in coal by ICP-MS after digestion using microwaveinduced combustion. Talanta, 2010, vol. 83, no. 2, pp. 364-369.

52. Ivanov V.V., Zarubina N.V., Zinoviev V.G., Okunev I.S., Shulyak G.I., Ostapenko D.S. Sravnitelnaya otsenka soderzhaniya reniya $\mathrm{v}$ uglerodsoderzhashchikh gornykh porodakh metodami mass-spektrometrii s induktivno svyazannoy plazmoy i instrumentalnogo neytronno-aktivatsionnogo analiza [Comparative assessment of rhenium content in carbon-containing rocks by inductively coupled plasma mass spectrometry and instrumental neutron activation analysis]. Voprosy atomnoy nauki i tekhniki. Seriya: Yaderno-reaktornye konstanty, 2015, Special Iss., pp. 49-58.

53. Ostapenko D.S., Zarubuna N.V., Ivanov V.V. Determination of rhenium in carbon-terigenous, organo-mineral and organogenic geological samples by ICP-MS method. $10^{\text {th }}$ International Symposium on Technetium and Rhenium - Science and Utilization. Moscow, Granica Publ. House, 2018. pp. 45-46.

54. Zarubina N.V., Ivanov V.V., Ostapenko D.S., Blokhin M.G., Panova E.G., Okunev I.S. Otsenka renienosnosti ugley metodom mass-spektrometrii s induktivno svyazannoy plazmoy [Evaluation of the retentivity of coals by inductively coupled plasma mass spectrometry]. Vserossiyskaya konferentsiya s mezhdunarodnym uchastiem [All-Russian conference with international participation]. Blagoveshchensk, AmurSC FEB RAS, IGiP FEB RAS, 2017. pp. 133-137.

55. Sorokin A.P., Konyushok A.A., Ageev O.A., Zarubina N.V., Ivanov V.V., Wang J. Distribution of rare earth and selected trace elements in combustion products of Yerkovetskoe brown coal deposit (Amur Region, Russia). Energy Exploration \& Exploitation, 2019, vol. 37, no. 6, pp. 1721-1736.

56. Seredin V.V. Metallonosnost ugley: usloviya formirovaniya i perspektivy osvoeniya [Metal content of coal: formation conditions and development prospects]. Ugolnaya baza Rossii. T. 6 [Coal base of Russia. V. 6]. Moscow, Geoinformmark Publ., 2004. pp. $453-519$.

57. Sedykh A.K. Kaynozoyskie riftogennye vpadiny Primorya (geologicheskoe stroenie, minerageniya $i$ geodinamika uglegeneza) [Cenozoic riftogenic depressions in Primorye (geological structure, minerageny and geodynamics of coal genesis)]. Vladivostok, Dal'nauka Publ., 2008. 248 p.

58. Sudyko A.F. Opredelenie urana, toriya, skandiya i nekotorykh redkozemelnykh elementov $\mathrm{v}$ dvadtsati chetyrekh standartnykh obraztsakh sravneniya instrumentalnym neytronno-aktivatsionnym metodom [Determination of uranium, thorium, scandium and some rare earth elements in twenty-four standard reference materials by the instrumental neutron activation method]. V Mezhdunarodnaya konferentsiya Radioaktivnost $i$ radioaktivnye elementy $v$ srede obitaniya cheloveka $\left[\mathrm{V}^{\text {th }}\right.$ International Conference on Radioactivity and Radioactive Elements in Environment]. Tomsk, STT Publ., 2016. pp. 620-624.

59. Dvorkin V.I. Metrologiya i obespechenie kachestva khimicheskogo analiza [Metrology and quality assurance in chemical analysis]. Moscow, Tekhnosfera Publ., 2019. 318 p.

60. OST 41-08-212-04. Upravlenie kachestvom analiticheskikh rabot. Normy pogreshnosti pri opredelenii khimicheskogo sostava mineralnogo syrya i klassifikatsiya metodik laboratornogo analiza po tochnosti rezultatov [Industrial Standard 41-08-212-04. Analytical quality management. Norms of error in determining the chemical composition of mineral raw materials and classification of laboratory analysis methods according to the accuracy of the results]. Moscow, VIMS Publ., 2004. 23 p.

61. Kuznetsova A.I., Petrov L.L., Finkelstein A.I., Menshikov V.I. Estimate of quality of elemental analysis of silicate rocks from results of participation in the international program of professional testing of geoanalytical laboratories - GEOPT. Analytics and Control, 2002, vol. 6, no. 5, pp. 584-592. In Rus.

62. OST 41-08-272-04. Upravlenie kachestvom analiticheskikh rabot. Metody geologicheskogo kontrolya kachestva analiticheskikh rabot [Industrial Standard 41-08-272-04. Analytical quality management. Methods for geological quality control of analytical work]. Moscow, VIMS Publ., 2004. 43 p.

Received: 24 February 2021.

\section{Information about the authors}

Natalya V. Zarubina, researcher, Far Eastern Geological Institute FEB RAS.

Maksim G. Blokhin, Cand. Sc., deputy director for science, Far Eastern Geological Institute FEB RAS.

Dmitry S. Ostapenko, junior researcher, Far Eastern Geological Institute FEB RAS.

Igor Yu. Chekryzhov, researcher, Far Eastern Geological Institute FEB RAS.

Sergey I. Arbuzov, Dr. Sc., professor, National Research Tomsk Polytechnic University.

Alexander F. Sudyko, senior researcher, National Research Tomsk Polytechnic University. 